\title{
Neurocristopathies: How New Discoveries in Neural Crest Research Changed our Understanding
}

\author{
Guillermo A Vega-Lopez ${ }^{1^{*}}$ and Manuel J Aybar ${ }^{1,2^{*}}$ \\ ${ }^{1}$ Institute of Biology "Dr. Francisco D. Barbieri", National University of Tucumán, Tucumán, Argentina \\ ${ }^{2}$ Higher Institute of Biological Research (INSIBIO, CONICET-UNT), Tucumán, Argentina
}

*Corresponding author: Guillermo A Vega-Lopez and Manuel J. Aybar, Faculty of Biochemistry, Chemistry and Pharmacy, Institute of Biology "Dr. Francisco D. Barbieri", National Universityof Tucumán, Tucumán, Argentina, Tel: 03814107214; E-mail: mjaybar@fbqf.unt.edu.ar; gavegalopez@fbqf.unt.edu.ar

Received date: September 26, 2018; Accepted date: October 25, 2018; Published date: November 4, 2018

Copyright: (c) 2018 Vega-Lopez GA, et al. This is an open-access article distributed under the terms of the Creative Commons Attribution License, which permits unrestricted use, distribution, and reproduction in any medium, provided the original author and source are credited.

\section{Introduction}

Neural Crest Cells (NCC) have long been recognized as the fourth layer of developing vertebrate embryos. The neural crest is a transient cell population that is probably heterogeneous but multipotent, giving rise to melanocytes, Schwann cells, sympathetic, parasympathetic and enteric neurons, enteric glia, endocrine cells, fibroblasts, muscle, bone, cartilage and meninges, among others cell types [1]. The disorders that stem from neural crest dysfunction, called Neurocristopathies (NCP) [2], are still only partially understood. Despite the great advances in our understanding of NCC formation and development, the causal link leading to NCP has remained elusive. In a recent review dealing with NCP [2] we provided a thorough analysis of $66 \mathrm{NCP}$ associated with a dozen "Cell Signaling Pathways", 4 different "families of Transcription Factors" and a wide diversity of "cellular processes". In over 5 model organisms (mouse, chicken, frog, fish and others, it has been demonstrated that NCP are linked to NCC faults during essential developmental processes. We also discussed the incorporation of new diseases or syndromes based on the defects of neural crest-derived tissues and organs that have also been unveiled very recently. In the light of recent discoveries we also included RASopathies, Ciliopathies, Ribosomopathies and defective epigenetic mechanisms as responsible for four newly established NCP categories.

\section{Developmental Insight}

Our recent review of NCP [2] also contributed to the understanding of the role of NCP in the development of different organs. A group of newly found neural crest derivatives were catalogued and described. Moreover, the recent advances in the field of neural crest developmental studies added to the review provide the basis for the proposal of a new general classification of NCP. This new classification, based on the axial origin of the neural crest derivatives, is more comprehensive and easier to understand and represents a guide to identify the possible origin of an NCP.

\section{The Making of Hematopoietic Precursors}

Our NCP review [2] also took into account an old proposal that considers hematopoietic precursors as derived from a common intermediate progenitor, the NCCs. This idea arose in part from the observation that hematopoietic precursors and their lymphoid progenitors are supported by the finding that the adult bone marrow microenvironmental niches are composed of various stromal cells, sympathetic non- myelinating glial Schwann cells and sympathetic nerve fibers. Since NCCs contribute to the development of all three, they are present in the human adult bone marrow to generate or regulate hematopoietic precursors $[3,4]$. The myelinating and non- myelinating Schwann cells of all adult nerves originate from the NC progenitors [5]. The deep revision of the current state on neural crest research still raises intriguing issues about the possible origin of hematopoietic precursors and other special cell types or tissues, and provides new hope that we may someday understand the anemia group of congenital abnormalities.

\section{Molecular Determinants of the Causes of NCP}

While several genetic and nongenetic factors have been linked to $\mathrm{NCP}$, the underlying molecular mechanisms that are disturbed during the development of NCC are not completely understood yet. As mentioned in the NCP review [2], this article can be thought of as an effort to value the essential research in the model organisms to increase the understanding of the molecular defects responsible for the causation of NCP. Therefore, identifying additional genes that also participate in the molecular mechanisms operating during normal development may uncover new candidate genes that can be added to genetic screenings for newborns at risk.

\section{Remaining Questions}

Probably the most important question is whether or not all the tissues and organs derived from the NCC have been discovered already. Another important issue is if all pathologies caused by the developmental failure of the NCC are currently known. The research for formally assessing or answering these questions is challenging in humans. Work on NCP [2] provides hints for new research as well as insights into the origin of these pathologies. One of the potential limitations in this field it is to find the appropriate organism to model the human disease of interest, and throughout its analysis to reach a better understanding of the molecular mechanism for the pathogenesis of NCP. It has been shown that the potential role for a gene suggested in one organism could be slightly different in another one (or in humans). More research with different model organisms could be essential to further pinpoint the molecular causes of NCP. Another potential problem is the step of extrapolating to human potential therapies that are successful in model organisms or human cell line cultures.

\section{Acknowledgments}

We are grateful to our colleagues for their helpful discussions. This publication was supported by grants from ANPCyT-Foncyt (to MJA PICT2013-1686 and PICT2015-1207; to GAVL, PICT2016-0835), and by grants from CIUNT (to MJA, PIUNT2018 26/D605). We especially thank Ms. Virginia Mendez for her proofreading. 
Citation: Vega-Lopez GA, Aybar MJ (2018) Neurocristopathies: How New Discoveries in Neural Crest Research Changed our Understanding. Cell Dev Biol 7: 195. doi:10.4172/2168-9296.1000195

Page 2 of 2

\section{References}

1. Pearse AG, Polak JM, Rost FW, Fontaine J, Le Lièvre C, et al. (1973) Demonstration of the neural crest origin of type I (APUD) cells in the avian carotid body, using a cytochemical marker system. Histochemie 34: 191-203.

2. Vega-Lopez GA, Cerrizuela S, Tribulo C, Aybar MJ (2018) Neurocristopathies: Newinsights 150 years after the neural crest discovery. Dev Biol pii: S0012-1606(17)30838-2.
3. Coste C, Neirinckx V, Sharma A, Agirman G, Rogister B, et al. (2017) Human bone marrow harbors cells with neural crest-associated characteristics like human adipose and dermis tissues. PLoS One 12: e0177962.

4. Jiang N, Chen M, Yang G, Xiang L, He L, et al. (2016) Hematopoietic stem cells in neural-crest derived bone marrow. Scientific Reports 6: 36411 .

5. Jessen KR, Mirsky R, Lloyd AC (2015) Schwann cells: Development and role in nerve repair. Cold Spring Harb Perspect Biol 7: a020487. 